\title{
An Analytical Framework for Vision Testing In Driving License Allocation in Nigeria
}

\author{
Adewale Olumide S. \\ Department of Computer \\ Science, Federal University of \\ Technology, Akure, Nigeria
}

\author{
Ogundele Oloruntoba S. \\ Department of Computer \\ Science, Federal University \\ Oye, Oye Ekiti, Nigeria
}

\author{
Iyare Otasowie \\ Department of Computer \\ Science, Federal University of \\ Technology, Akure, Nigeria
}

\begin{abstract}
Allocation of Driving Licensing in Nigeria in the past was done haphazardly and as a result drivers with poor driving culture were usually allocated driving license. This probably was followed by unprecedented wave of road traffic accidents with attendant human and material losses. In other to reduce these losses, the federal road safety commission at its inception introduced some measures to curb the issue of licensing. This work, in line with the technological trend proposed an automated licensing scheme to improve on the efficiency of the previous system. The system was tested with 50 applicants and the result presented.

Keywords:
\end{abstract}

\section{General Terms}

Analytical Framework, Computer Vision

\section{Keywords}

Computer Vision, License, Database Management System.

\section{INTRODUCTION}

Before the creation of the Federal Road Safety Commision (FRSC) in 1988, there was no provision of how to cater for safety on Nigeria Highways and also the approach to road safety was handled unilaterally in different quarters [1]. The traffic situation in the country then was characterised as chaotic, unpredictable and indeed dangerous. This resulted in unprecedented wave of road traffic accidents with attendant human and material losses. Within this era, public awareness and interest in road safety was minimal, uncoordinated and haphazard resulting in granting license to drivers who lack good driving culture. According to [2], policies and concerted efforts at enforcing regulations were lacking and as a result the road traffic accidents recorded at that time were 302 at 16 deaths per 1,000 vehicles [3].

In Nigeria, the road traffic situation is of concern to the entire populace as good road network, that match the basic environmental and mechanical conditions are not readily available [4]. As a result, road traffic accident formed part of the major causes of death and injuries in the country.

The following are have been identified as major factors responsible for poor driving culture in Nigeria:

- Informal training method

- Lack of basic knowledge of traffic rules and regulations

- Driving for economic gains

- Overconfidence as a result of improper measurement of self driving ability

- Impatience leading to flagrant violation of traffic rules, most times resulting in driving against the traffic

- Crude or manual vision test system
- Others are road and driving environment

The end result of these factors is accident, which leads to injuries, death and loss of valuables. It was against this backdrop that the Federal Road Safety Commission was created on 18th February, 1988 by the FRSC Act Cap 141. The Commission adopted an integrated approach such as: enforcment, public education and research, involvement of volunteers and youths, injection of order and control into licensing of drivers and vehicles, provision of cultural related highway code and traffic rules, streamlining and standardisation of traffic related matters, creation and promotion of road safe culture [5]. The significant impact of the FRSC is in the reduction of road traffic accident casuality rate.

In the course of the operation of the FRSC, it was discovered that the carnage on our roads had its route in remote factors like training and licensing of drivers. Apprehension of offending drivers was difficult due to the uncoordinated fashion in which licensing and documentation were done. In order to enhance cross-country vehicular traffic, a nationally controlled system was developed to achieve the following:

- Design the drivers' licences to be used by the various caregories of vehicle operators.

- Determine the requirements to be satisfied by an applicant for a drivers' licence.

- Design vehicle number plate identification.

Following the above, the Commission instituted a harmonisation of the various road traffic laws into the National Road Traffic Regulations. In order to achieve excellence, administrative steps were put in place to forestall untoward developments capable of impinging on the system. These steps include:

i Fresh applicants shall include those still in possession of the old driver's licence and those undergoing driving tests for the first time.

ii All testing procedures including vision test as required by the law must be carried out before a driver's licence can be issued

iii Successful applicants must complete, in triplicates, the form $18 \mathrm{~N}$ for National Driver's Licence (fresh) obtained from any Motor Licensing Office throughout the Federation on the payment of prescribed fees.

iv A copy is to be returned to FRSC through the Motor Licensing Office while the Motor Licensing Authority and the Vehicle Inspection Officers retain a copy each. The original copy of the learner's permit approved and duly signed by the Vehicle Inspection Officer is attached to the form.

$\mathrm{v} \quad$ Renewal of driver's licence, is done at the licensing office after payment of prescribed fees. The forms are processed and printed licences are returned to 
the licensing office (MLA). However an applicant whose licence has expired for more than two years should be certified by the Road Traffic Office before renewal is granted.

There are nine classes/categories as indicated in table 1 .

Table 1: Class categories table

\begin{tabular}{|l|l|l|}
\hline S/N & CLASS & MEANING \\
\hline 1. & Class A & Motor Cycle \\
\hline 2. & Class B & $\begin{array}{l}\text { Motor vehicle less than 3 tones gross weight other than } \\
\text { motor cycle, taxi stage carriage or omnibus. }\end{array}$ \\
\hline 3. & Class C & $\begin{array}{l}\text { Motor vehicle less than 3 tones gross weight other than } \\
\text { motor cycle. }\end{array}$ \\
\hline 4. & Class D & $\begin{array}{l}\text { Motor vehicle other than motor cycle, taxi stage carriage or } \\
\text { omnibus but excluding an articulated vehicle or vehicle } \\
\text { drawing a trailer. }\end{array}$ \\
\hline 5. & Class E & Motor vehicle other than motor cycle or articulated vehicle. \\
\hline 6. & Class F & Agricultural machines and tractors. \\
\hline 7. & Class G & Articulated vehicle. \\
\hline 8. & Class H & Earth moving vehicle. \\
\hline 9. & Class J & Special for physically handicapped. \\
\hline
\end{tabular}

The drivers license has today gone beyond usage in relation to motor traffic regulation only. It has come to be seen as a major cause of reliable identity in the financial sector and for Nigerians travelling abroad. This is simply because of the secured features (like the signature of holders, thumb print of applicant) impeded into it and the ease at which confirmation can be made at FRSC [5]

\section{REVIEW OF EXISTING WORKS}

Vision is the fundamental way we perceive and respond to stimuli all around us [6]. It involves sight, knowledge and our reaction to things. Vision according to the American Automobiles Association is estimated to account for 85 to 95\% of all sensing clues in driving. Vision loss can occur when sight weakens with usually with increasing age. Low vision results from congenital, genetic, or acquirable factors. Vision impairment occurs when glasses, contact lenses, or surgery cannot correct the vision loss. Cataracts, Age-related Macular Degeneration (AMD), glaucoma, and diabetic retinopathy account for the four main causes of visual impairment and blindness in the United States [7]. [8] indicate that visual impairment may be caused by both systemic conditions and specific eye conditions. A study conducted by [6] highlighted the importance of the use of automated testing techniques and the significance of vision screening measures other than standard visual acuity testing for assessing all drivers and, in particular, at-risk drivers and older drivers. The study demonstrated that environmental factors and manner of collisions increase in accident involving drivers between ages 50 to 59 years in both Arizona and Florida. Their findings span a period of 11years (1991 to 2001) but the study, however, did not consider all the visual parameters.
Statistical modeling adopted in [9] for vision testing revealed that Snellen acuity of 6/9-2 has an equivalent failure rate to the number plate test. They found that those with a binocular visual acuity of $6 / 7.5$ had a $99 \%$ chance of passing the test, whereas those seeing $6 / 18$ binocularly had only a $6 \%$ chance of passing. The model was based on a population of only 28 subjects; the pre-2001 number plate design was used. A study by [10] using the old number plate design, found out that the binocular visual acuity of those who only just read the number plate varied from $6 / 9+2$ to $6 / 12-2$, with an average of $6 / 10$. The work reported that $26 \%$ with a binocular VA of $6 / 9$ failed to correctly read the number plate and $34 \%$ of those with a binocular VA of $6 / 12$ passed. Although this study has its limitations, these pass/fail percentages should be noted carefully since they highlight the problem we face advising patients based on their binocular Snellen acuity alone. The work of [11] was developed to explore ways in which the International Council of Ophthalmology (ICO) could contribute towards the global harmonization issue in the area of Visual assessment for driving license issuance. In the work, sensitive issues were raised and possible solution was presented. In [12], a study with 2520 participants was conducted to determine the role of vision and visual attention factors in automobile crash involvement. Of the 2520 participants in the Study, 1801 were eligible drivers for the analysis. The remaining 719 were not eligible. However, the study was limited only to Salisbury, Maryland while the findings may not generalize to larger cities with more dense populations. The visual requirements for obtaining a driver's license vary from state to state. Some states screen only first time applicants while others require an assessment each time a driver's license is renewed. The later instance is becoming less the norm as more states move to periodic renewal by mail. Few states require color and/or depth perception tests but use the results for "advisory" purposes only rather than as a basis for denying licensure. The most common acuity requirement for unrestricted licensure is 20/40 [13]. More rigorous visual requirements are imposed upon commercial driver's license applicants - but few of these drivers are aged 65 or older. The 20/40 visual acuity standard has evolved by consensus. Part of the basis for accepting this value is that in the whole population, 20/40 acuity represents an extreme level of visual loss. That is, at least $97 \%$ of the general population can be corrected to 20/40 acuity or better [14]. Ironically, the 20/40 acuity standard does not ensure a good match between the abilities of the licensed driver and the design criteria used to build the nation's highways. For example, current standards for the design of highway signs assume a visual acuity of $20 / 23$ (i.e. the $50: 1$ rule $-50 \mathrm{ft}$ of visibility is afforded by every 1 in text legend height). Some investigators have argued that the 20/40 acuity standard is far too restrictive. It is difficult to determine the high-contrast resolution needs for driving because, apart from signs, most spatial information in the visual tasks of driving is at medium or low contrast. Although the 20/40 acuity criterion segregates only around $3 \%$ of the general driving population, the vast majority of those affected are elderly. According to the Framingham Study, approximately $13 \%$ of those between the ages of 75 and 85 have best corrected visual acuities which are worse than the 20/40 standard. In fact, fully half of this same group is estimated to be legally blind (i.e., 20/200 or worse). Little epidemiological data is available which describe the visual capacity of those older than 85 - although [15] estimated that the incidence of severe visual deficit among the old is on the order of $33 \%$ or higher. 


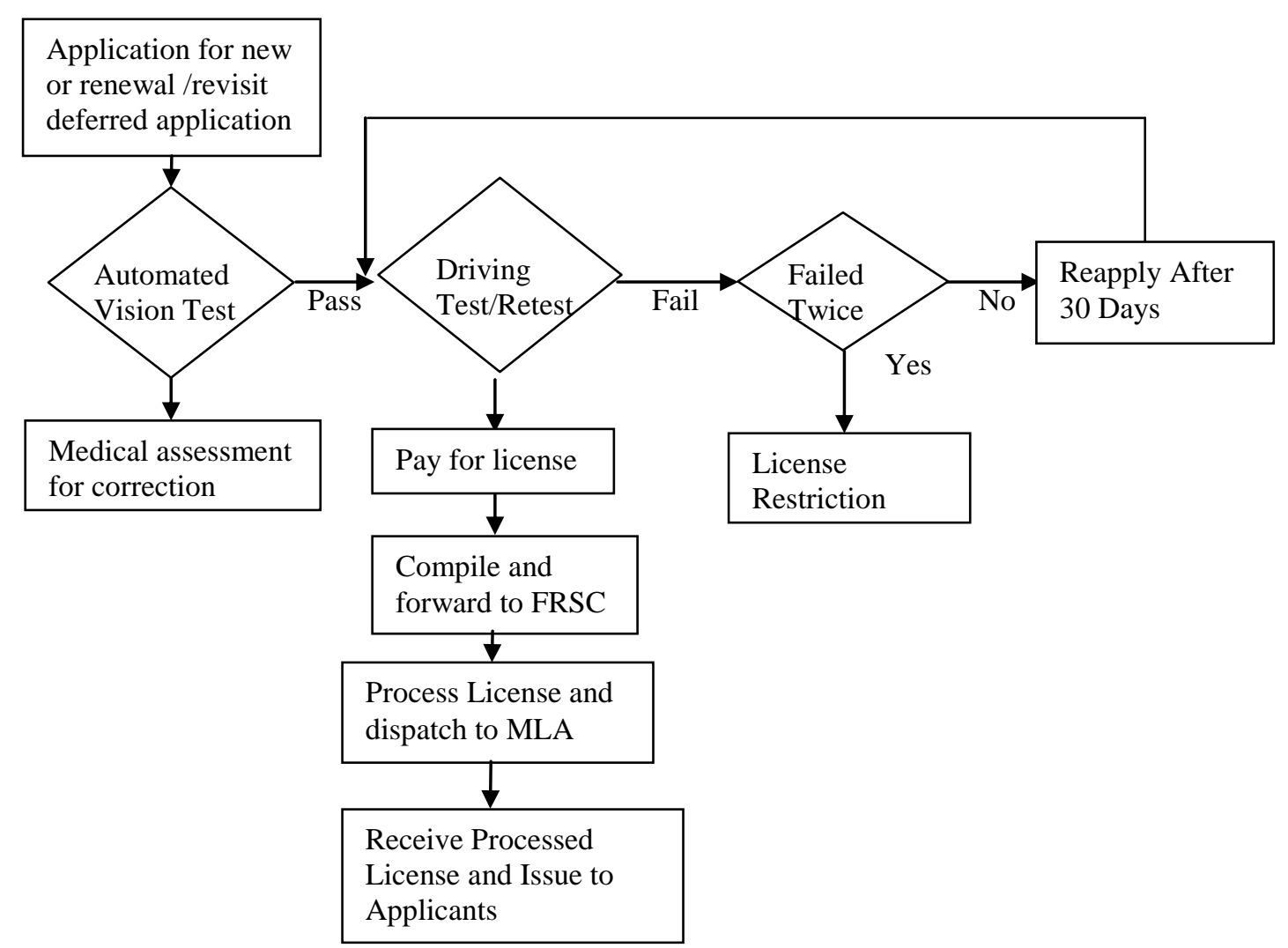

Fig.1: Flowchart of drivers licence issuance

\section{SYSTEM DESIGN}

Seven main vision tests are used in this system. These are letters (alphabets), compass direction arrow (NESW - North East South West) with 1 and 2 pixels respectively, number on four corners with 1,2 and 3 pixels respectively and missing boxes. Furthermore, each test contains ten different objects. These objects are chosen randomly for each of the tests. Each applicant is expected to type the test objects into the blank boxes provided.

The classes of the driver's license are 9 (as shown in table 1); Let the classes of driver's license be $C_{k}$ where $K=\{1,2, \ldots \ldots \ldots . . .9\}$

Applicant A can only apply for renewal or new driver's license within the class $C_{k}$. Thus let $A_{k}$ represent the application of applicant $\mathrm{A}$.

The applicant by standard goes through seven different automated vision tests $V_{i}$ where

$i=\{1,2, \ldots 7\}$ i.e. the number of vision test and

And automated test has ten test objects $O_{j}$

$j=\{1,2, \ldots 10\}$ i.e. the number of test objects.

Therefore, to compute the total score from both the vision and object tests; we have

$\operatorname{Score}_{i j}=\sum_{i=1}^{n} V_{i}+\sum_{j=1}^{m} O_{j}$

Thus

$$
\operatorname{Score}_{i j}=\sum_{i, j=1}^{n, m}\left(V_{i}, O_{j}\right) ; n=7, m=10
$$

And

$A_{k}\left\{\begin{array}{ccc}\text { Successful } & \text { if } \text { Score }_{i j} \geq 50 \% \\ \text { Not Successful } & : & \\ & \text { otherwise }\end{array}\right.$

If $A_{k}$ is not successful then medical assessment test for corrections is advised, otherwise $A_{k}$ is moved on to the next stage of driving test with VIO (Vehicle Inspection Officer). The VIO test is a function of set conditions given as follows;

Let $V I O_{\text {test }}(R)=\left\{\begin{array}{l}1 \\ 0\end{array}\right.$

Then $V I O_{\text {test }}(R)=f\left(\right.$ Stated_Conditions $\left.{ }_{Z}\right)$

where $Z=9$;

If $V_{\text {I }} O_{\text {test }}(R)=1$, the applicant is issued a driving licence subject to test $=\left\{C_{k}\right\}$ and

elseif $\operatorname{VIO}_{\text {test }}(R)=0$, application unsuccessful

Therefore $V I O_{\text {test }(R)} \rightarrow\left\{C_{k}\right\}$, (one to many mapping) and the applicant is issued with the license based on the analysis below or decline otherwise: 
$A_{k(\text { issued })}=\left\{\begin{array}{c}\text { Class } A . . J, \text { if } V I O_{\text {test }}(R)=1 \\ 0, \quad V I O_{\text {test }}(R)=0\end{array}\right.$

If $A_{k \text { (issued })}=1$, then the application for licence will be process further otherwise the applicant reapplies after 30days the first time, and subsequent failure, a restriction of until six months after. The flow is shown in fig.1.

\subsection{Technological Approach}

The technological approach for the development of the Automated Driver's Licence vision test system is based on AMP (Apache, MySQL, and PHP) open source solution [16]. This system is built around a three-tier architecture model [17]. This is shown in Fig. 2. At the base of an application is the database tier, consisting of the database management system that manages the database containing the data users create, delete, modify, and query. MySQL relational database is used to provide the required functionality.

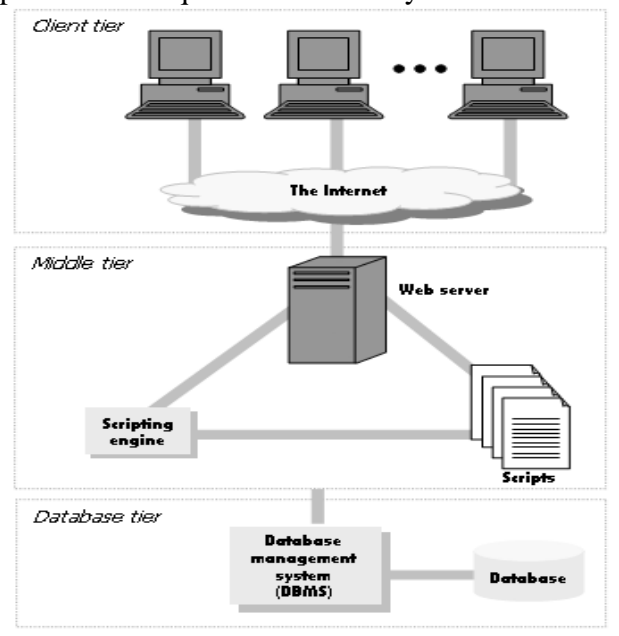

Fig. 2: Three-tier Architecture Model

The general form of a relation is given by $R\left[A_{1}, A_{2}, \ldots, A_{k+1}\right.$, $\left.\ldots, A_{n-1}, A_{n}\right]$. The name of the relation is represented by $R$, the set $\left\{A_{i, i}=1,2, \ldots, n\right\}$ represents the attributes of the relation $R$. The database objects used in the automated vision test system are:

applicant[class_of_licence, name, address, sex, date_of_birth, height, glasses__ requirement, signature, thumbprint, passport_photograph, blood_group]

drivers_licence[driver's_licence_no, test_centre_ no, date_of_test, time_of_test, date_of_issue, state_ of_issue, expiry_date]

driving_test[applicant_no, name, address, sex, class_of_licence, examiner_name, examiner_no, result_of_driving_test]

driving_licence_fee [class_of_licence,fee]

driving_test_centre[test_centre_no, test_centre_address]

driving_test_examiner[examiner_licence_no,

examiner_name,examiner_address, test_centre_no]

vision_test[applicant_no,applicant_name, applicant_address, letters,compass_direction_arrow, numbers_on_four_corners, missing_boxes]
Built on top of the database tier is the complex middle tier, which contains most of the application logic and communicates data between the other tiers. The web server is apache and it is running under Windows operating system which is used to achieve a secured client-server communication.

Entry point to some of the automated vision test systemm requires users' authentication and authorization because of the sensitivity of the admission process. Since users access the system remotely, therefore a built-in security system forces users to login first. There are four main elements that made up the system user authentication module: user registration, login and logout, changing password and resetting passwords. A valid user or admission officer could perform each of these elements. To register a user, user supplies his user identification number, and the preferred username, password and password confirmation via an html form, and if the user identification number is found in the user table and both password and password confirmation are the same, it then registers the username and password in the user table. Registered users can now login by supplying his username and password details into an html form and submit it, the entries will be processed and the user will be logged on if the authentication is successful. Users are also allowed to change their passwords and in addition to this, the system also deals with the common situation in which a user has forgotten his password.

\section{RESULT AND DISCUSSION}

In the automated vision test system, an applicant will perfom different tests as shown below (figures 3 to 6 )
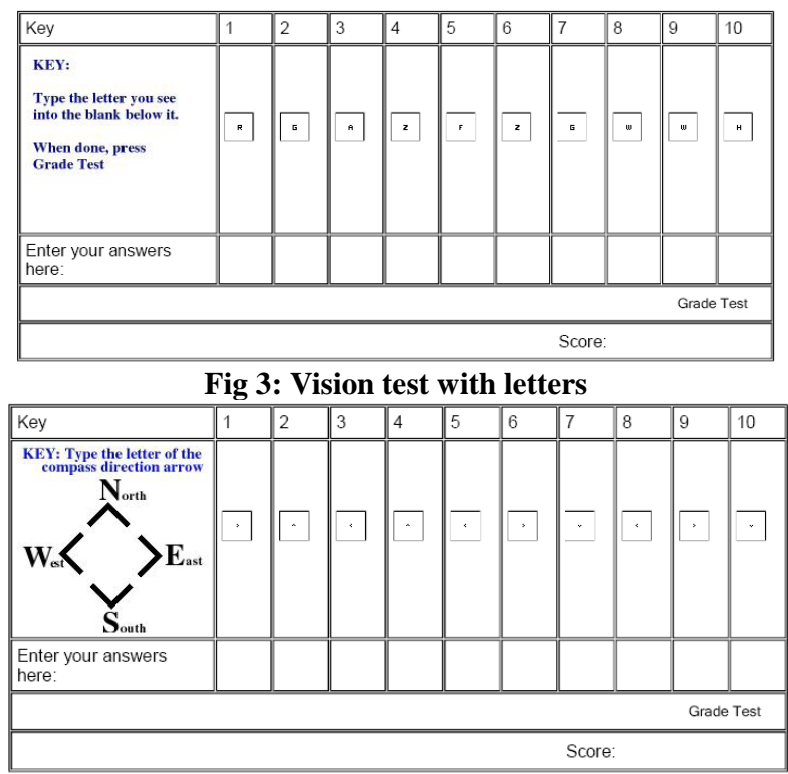

Fig 4: Compass direction arrow

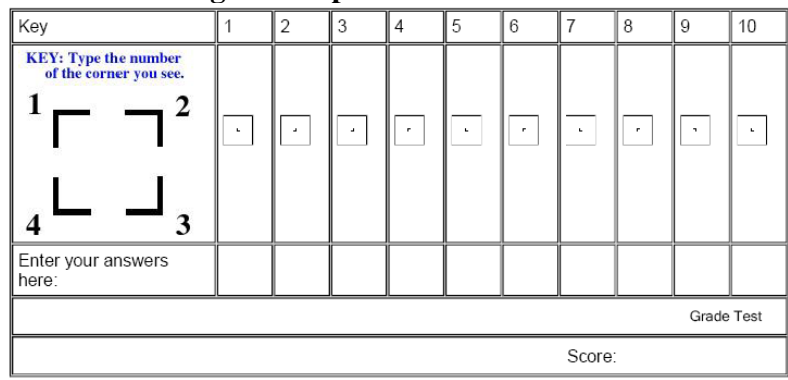

Fig 5: Number on four corners 


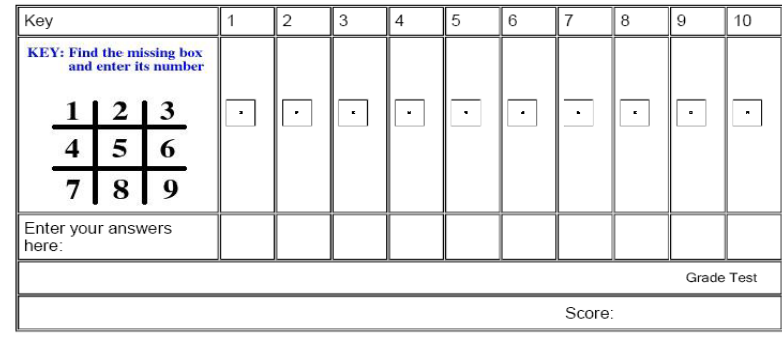

Fig 6: Missing boxes

The results of the tests are graded. If the applicant scores less than 50 percent, the applicant will be refer for medical assessment for correction. On the other hand if he scores 50 percent and above, then the applicant proceeds to Vehicle Inspection Office (VIO) for driving test.

An applicant who passes the driving test will subsequently require to complete either Form RS18N for fresh applicant or Form RS15R for renewal, after when such applicant is authorize to pay the necessary fees for driver's licence processing. However, if he fails, the applicant reapplys after 30 days. An applicant who fails twice will be restricted for six months. The system was simulated with 50 applicants at random. The vision test result revealed that $37(74 \%)$ of the applicants were recommended for the VIO driving test while the remaining $13(26 \%)$ applicants were referred to be assessed medically. From the result of the VIO, 26 (70.3\%) of the applicants passed and were asked to proceed with the license issuance while 11 (29.7\%) failed the driving test and were asked to retake the test after 30days.

\section{CONCLUSION}

More vehicles are being bought daily and the number of drivers requiring license are now on the increase. Based on these trends, there is the likelihood of a surge in the number of licensed drivers over the next 10 years. These changes may also include a significant rise in the number of collisions, injuries, and fatalities among drivers unless action is taken now to avert collision related crashes. As the Nigeria population significantly increases over the next 30 years, there is need for more effective driver's license screening tools. These issues need to be addressed in each state and country now as the driver population grows and necessitates adequate accommodations to improve safe driving.

This automated vision test framework is therefore recommended for adoption and implementation. It will provide a significant improvement over the current system, especially for screening the vision of all driver's licence applicants and renewals. Also the provision of retesting of disqualified drivers before regaining their licences will go a long way in reducing collision, injury, and fatality risks and the staggering costs associated with these events as they affect drivers of all ages [18].

\section{REFERENCES}

[1] J. A. Odeleye. 2000. Improved road traffic environment for better child safety in Nigeria. ICTCT workshop, $13^{\text {th }}$ Int. Conf. On Pedestrians and Road Design, eds.. pp209218.

[2] M. Wiklund, L. Simonsson, A. Forsman, O. Hallberg, and O. Johansson, 2011. Traffic safety and the economic situation; Model approaches and a literature survey". VTI SE-581 95 Linköping Sweden.

[3] B.O. Oyeyemi, 2003a. Strands in road traffic administration in Nigeria". Clemeve Media Konsult
[4] E.P. Osawe, G. Osawe, 2013. Road safety and security on african roads, nigeria road as a case study". Injury prevention safety abstract, 2010. Available at: injuryprevention.bmj.com, downloaded on June 2, 2013.

[5] B.O. Oyeyemi, 2003b. Productivity and road traffic administration in Nigeria. Issues, Impedements and Strategy. Clemeve Media Konsult

[6] ESRA Consulting Corporation. 2005. New, Improved, Comprehensive, and Automated Driver's License Test and Vision Screening System

[7] M. Desai, L. Pratt, H. Lentzner, and K. Robinson. 2001. Trends in vision and hearing among older americans. Aging Trends; No, 2. Hyattsville, Maryland: National Center for Health Statistics. 2001.

[8] J. Torpey, M., Lynm, G. Cassio, and M. Richard. 2003 "Causes of visual impairment." Journal of the American Medical Association 290:2088.

[9] Drasdo N and Haggerty CM. 1981. A comparison of the British number plate and Snellen vision tests for car drivers. . Ophthalmic and Physiological Optics; 1: 39-54

[10] Currie Z, Bhan A and Pepper I. 2000. "Reliability of Snellen charts for testing visual acuity for driving: prospective study and postal questionnaire" British Medical Journal; 321: 990-2

[11] August Colenbrander and Jean-Jacques De Laey. 2006. Vision Requirements for Driving Safety, with emphasis on Individual Assessment. International Council of Ophthalmology at the $30^{\text {th }}$ World Ophthalmology Congress Sao Paulo, Brazil, February 2006.

[12] Rubin G.S, Edmond S.W, Karen B.R, Keyl P.M, Freeman E.E, West S.K, and SEE Project Team. 2007. A Prospective, Population-Based Study of the Role of Visual Impairment in Motor Vehicle Crashes among Older Drivers: The SEE Study

[13] National Eye Institute, National Institute of Health. "Vision loss from eye diseases will increase as americans age. National Eye Institute, National Institute of Health: U.S. Department of Health and Human Services. [Cited 15 April 2004]. Available from http://www.nei.nih.gov/news/pressreleases/041204.htm

[14] American Automobile Association. 1991. Foundation for traffic safety. Washington, DC: American Automobile Association (AAA) Publications.

[15] Congdon, N. G., D. S. Friedman, and T. Lietman. 2003. Important causes of visual impairment in the world today. The Journal of the American Medical Association(JAMA) 290(15):2057-60.

[16] O.S. Adewale, 2006. University digital libraries: an initiative for teaching, research and service. Adeyemo publishing house.

[17] O.S. Adewale, 2006. University digital libraries: adaptive personalised e-learning system. Published by yerimah prints,

[18] Department of Transport. 2011. Strategic framework for road safety. Crown, London. Pp 1-75. 